Research report

\title{
Weight management, psychological distress and binge eating in obesity. A reappraisal of the problem ${ }^{\text {is }}$
}

\author{
Riccardo Dalle Grave ${ }^{\mathrm{a}}$, Simona Calugi ${ }^{\mathrm{a}}$, Maria Letizia Petroni ${ }^{\mathrm{b}}$, Silvia Di Domizio ${ }^{\mathrm{c}}$, Giulio Marchesini ${ }^{\mathrm{c}, *}$ \\ the QUOVADIS Study Group \\ a Department of Eating and Weight Disorder, Villa Garda Hospital, Via Montebaldo 89, I-37016 Garda (VR), Italy \\ ${ }^{\mathrm{b}}$ Unit of Nutrition Rehabilitation, San Giuseppe Hospital, Via Cadorna 90, I-28824 Piancavallo (VB), Italy \\ ' Clinical Dietetics, "Alma Mater Studiorum" University, Via Massarenti 9, I-40138 Bologna, Italy
}

\section{A R T I C L E I N F O}

\section{Article history:}

Received 2 January 2009

Received in revised form 1 November 2009

Accepted 20 November 2009

\section{Keywords:}

Weight loss

Depression

Binge eating

Psychological distress

Obesity treatment

\begin{abstract}
A B S T R A C T
The psychological effects of dieting and weight loss have been an area of controversy in obesity. As part of a large multicenter study involving 1944 obese subjects seeking treatment at Italian medical centers, we investigated the effects of weight loss on psychological distress and binge eating in 500 subjects remaining in continuous treatment at different centers with slightly different strategies (78.8\% females; age: $M=46.2$ years, $\mathrm{SD}=10.8$; $\left.\mathrm{BMI}: M=37.3 \mathrm{~kg} / \mathrm{m}^{2}, \mathrm{SD}=5.6\right)$. At baseline and after $12 \mathrm{months}$ all subjects were evaluated by the Symptom CheckList-90 Global Severity Index (SCL-GSI) and by the Binge Eating Scale (BES). In both males and females, weight loss was associated with improved psychometric testing. Changes in SCL-GSI were associated with changes in BMI $(\beta=0.13 ; t=2.85 ; p<0.005)$, after adjustment for age, gender, initial BMI and center variability. Similarly, BES changes were associated with BMI change $(\beta=0.15 ; t=3.21 ; p<0.001)$. We conclude that in subjects compliant to follow-up a successful management of obesity, not directly addressing psychological distress, is associated with a significant improvement of both psychological distress and binge eating, linearly related to the amount of weight loss, independently of treatment procedures.
\end{abstract}

(c) 2009 Elsevier Ltd. All rights reserved.

\section{Introduction}

The psychological effects of dieting and weight loss have been a matter of controversy in the field of obesity management. A review of several early studies (before the 1970s) described negative emotional consequences to dieting (Stunkard \& Rush, 1974), whereas later studies found an improvement or no changes in the symptoms of depression and anxiety in obese patients treated by behavior modification combined with moderate calorie restriction (Bryan \& Tiggemann, 2001; Taylor, Ferguson, \& Reading, 1978; Wadden \& Stunkard, 1986; Wing, Blair, Marcus, Epstein, \& Harvey, 1994; Wing, Marcus, Epstein, \& Kupfer, 1983), severe calorie restriction (Wadden, Foster, \& Letizia, 1994; Wadden, Stunkard, Brownell, \& Day, 1985; Wing, Marcus, Blair, \& Burton, 1991), or use of weight loss drugs (Brownell \& Stunkard, 1981; Kiortsis, Tsouli, Filippatos, Konitsiotis, \& Elisaf, 2008; Wadden et al., 1997). Two

\footnotetext{
A complete list of participants in the QUOVADIS study has been previously published (Diab Nutr Metab, 2003;16:115-24).

* Corresponding author at: Clinical Dietetics, “Alma Mater" University of Bologna, Policlinico S. Orsola, Via Massarenti 9, I-40138 Bologna, Italy.

E-mail address: giulio.marchesini@unibo.it (G. Marchesini).
}

systematic reviews concluded that professional weight loss interventions are not associated with negative psychological consequences and eating disorders, but rather with a modest improvement in psychological status both in obese adults (National Task Force on the Prevention and Treatment of Obesity, 2000) and in overweight children and adolescents (Butryn \& Wadden, 2005). Finally, a recent study found that the sole control of food intake as dietary treatment for obesity, not accompanied by any intervention on psychological distress, improved eating psychopathology, self-esteem and mood (Werrij, Mulkens, Hospers, Smits-de Bruyn, \& Jansen, 2008). These conflicting results may be explained by patients' selection, with earlier studies including patients with emotional disturbances treated in psychiatric settings, and more recent studies including patients generally free of clinical depression (National Task Force on the Prevention and Treatment of Obesity, 2000).

Several studies also assessed the effect of weight loss treatment on binge eating. Behavioral weight loss treatment combined with moderate energy restriction was associated with fewer episodes of binge eating in patients with binge eating disorder (Agras et al., 1994; Porzelius, Houston, Smith, Arkin, \& Fisher, 1995; Wing, Marcus, Epstein, Blair, \& Burton, 1989) and no onset of binge eating in those who, before treatment, were not binge eaters (Porzelius 
et al., 1995; Sherwood, Jeffery, \& Wing, 1999; Wadden et al., 1994, 2004). Similar outcomes on binge eating were observed with severe energy restriction (Raymond, de Zwaan, Mitchell, Ackard, \& Thuras, 2002; Wadden et al., 1994; Yanovski, Gormally, Leser, Gwirtsman, \& Yanovski, 1994), but one study reported a worsening of binge eating after 12 weeks' consumption of a liquid very lowcalorie diet (Telch \& Agras, 1993). A significant reduction in the frequency of binging episodes was also reported with the use of weight loss drugs, associated with moderate energy restriction (Golay et al., 2005; Wilfley et al., 2008). A recent experimental study showed that nonobese women randomly assigned to receive a low-calorie diet significantly decreased bulimic symptoms in comparison to subjects assigned to a waitlist control condition (Presnell \& Stice, 2003), an outcome confirmed also by a longitudinal study (Stice, Martinez, Presnell, \& Groesz, 2006) and a randomized efficacy trial (Stice, Marti, Spoor, Presnell, \& Shaw, 2008). These data suggest that successful dietary restraint tends to curb bulimic symptoms.

In turn, the influence of binge eating on weight loss has been extensively investigated. In general, pre-treatment binge eating status was not associated with weight loss outcomes (Delinsky, Latner, \& Wilson, 2006; Teixeira, Going, Sardinha, \& Lohman, 2005), but changes in eating patterns during treatment were rarely examined. In the Look AHEAD (Action for Health in Diabetes), a large trial examining the long-term effect of intentional weight loss on cardiovascular disease in overweight and obese adults with type 2 diabetes, the participants who stopped binge eating were just as successful at weight loss as non-binge eaters after 1 year, and lost more weight than those who continued or started binging during treatment (Gorin et al., 2008). A review of the literature reported an association between the amelioration of binge eating induced by specific treatment and weight loss (Yanovski, 2003). In summary, available data indicate that baseline binge eating does not obstacle weight loss.

Most data on the psychological effects of weight loss come from well-defined research settings. These studies have several strengths (e.g., internal validity/rigorous research design, low attrition rates, standardized interventions), but limited external validity because of exclusion criteria and specific procedures to reduce attrition (e.g., incentives for patients who participate in follow-up). We analyzed the effect of weight loss on psychological outcomes in obese patients participating in a large multi-site Italian observational study (Melchionda et al., 2003). Based on previous findings in research settings, our main hypothesis was that weight loss achieved with treatments not specifically addressing psychological distress and binge eating was associated with improved psychological outcomes also in patients observed in the "real world" of obesity centers.

\section{Materials and methods}

\section{QUOVADIS study planning and protocol}

The QUOVADIS (QUality of life in Obesity: eVAluation and DIsease Surveillance) study planning and protocol have been described in details in a previous paper (Melchionda et al., 2003). QUOVADIS is a purely observational study on quality of life, psychological distress, and eating behavior in obese patients seeking treatment at 25 obesity medical centers accredited by the Italian Health Service and treated according to their specific protocols. All the centers adopted an eclectic approach including different forms of dieting and physical exercise, combined in some cases with basic behavior modification strategies (e.g., food diary), drugs and bariatric surgery (less than $2 \%$ of patients). While the theory on which the treatment was based varied between centers, all adopted an initial intensive treatment period (3-6 months), followed by a less intensive continuous care (a follow-up control every 2-4 months) with periodic controls for an indefinite period of time (Dalle Grave et al., 2005).

All individuals with obesity (BMI $\geq 30 \mathrm{~kg} / \mathrm{m}^{2}$ ), consecutively seeking treatment, including those with binge eating, were eligible for the study, provided they were not on active treatment at the time of enrollment, were in the age range between 25 and 65 years and agreed to complete a package of self-administered questionnaires. To ensure that subjects were truly consecutive the medical record number of the patients was monitored across sites.

A systematic medical and psychosocial evaluation of participants was planned at baseline, approximately 1 week before the beginning of treatment, and after 12 months. Seven centers refused to collect the 12-month follow-up data because of limited human resources and longitudinal data were thus available only for 18 centers. For the purposes of the present report, 6 cases who underwent bariatric surgery were excluded from analysis.

The protocol was approved by the ethical committees of the individual centers, after approval by the ethical committee of the coordinating center (Azienda Ospedaliera di Bologna, Policlinico S. Orsola - Malpighi). All participants gave written informed consent for participation.

\section{Measures}

\section{Case Report Form}

The Case Report Form was filled in by physicians at the time of enrollment by directly interviewing patients.

\section{Psychosocial measures}

At baseline and at follow-up, the participants completed a battery of questionnaires measuring psychological distress and binge eating.

The Symptom CheckList-90R (SCL) (Derogatis \& Cleary, 1977) was used to identify psychological distress. For each item, patients scored how much that problem has distressed them during the previous week, with responses ranging from 0 (not at all) to 4 (extremely). The 90 items of the test were used to compute the general symptom index (GSI), which is an indicator of the overall psychological distress (Derogatis \& Cleary, 1977). A value $\geq 1$ in SCL-GSI is suggestive of psychopathology (1.00-1.49, mild; $1.50-$ 1.99 , moderate; $\geq 2.00$, severe) (Derogatis \& Cleary, 1977). The various subscales of SCL were not considered for the purposes of the present study.

The Binge Eating Scale (BES) (Gormally, Block, Daston, \& Rardin, 1982) was used to measure the severity of binge eating. It examines both behavioral signs (eating large amounts of food) and feeling or cognition during a binge episode (loss of control, guilt, fear of being unable to stop eating) in 16 items. Scores $\geq 27$ conventionally serve as a cutoff value for identifying the presence of severe binge eating and $\leq 16$ as a cutoff value for mild or no binge eating (Greeno, Marcus, \& Wing, 1995).

\section{Weight and height}

Weight was measured on a medical-balance and height by a stadiometer in patients with underwear and no shoes. Weight change was examined from baseline to 12 months.

\section{Statistical analyses}

All weight data (in $\mathrm{kg}$ ) were transformed into BMI units to improve comparison between genders. A first descriptive analysis was used to obtain a qualitative evaluation of clinical data, response to questionnaires and patients' outcomes. Changes in clinical parameters at 12 months were tested for significance by means of parametric and non-parametric tests for paired data, and 
were transformed into effect sizes for an immediate assessment of their magnitude (Cohen, 1977; Kazis, Anderson, \& Meenan, 1989). Differences between completers and drop-outs were carried out using $t$-test for independent samples.

A $2 \times 2$ ANOVA was performed to compare pre- vs. posttreatment values (within group factor) and females vs. males (between group factor). Pearson's correlation was used to show the correlations between relevant variables.

Multiple regression analysis was used to test the association between change in BMI and changes in SCL-GSI (or BES) at 12month follow-up in two separate models. $\triangle$ SCL-GSI was the dependent variable in Model 1, where change in BMI was the independent variable. This model was adjusted for gender, initial BMI, age and center variability, coded as dummy variable to identify the different treatment centers. In a second regression analysis, the Model 1 was further adjusted for basal and 12-month changes in BES. $\triangle$ BES was the dependent variable in Model 2, with changes in BMI as the independent variable. Also this model was adjusted for gender, initial BMI, age and center variability, and further adjusted for basal and 12-month changes in SCL-GSI in a second regression analysis.

The Variance Inflation Factor was calculated to assess correlation between predictor variables and to exclude multicolinearity.

Data are reported as mean \pm standard deviation (SD) or percentage. All analyses were performed using SPSS for Windows, Version 15.0.

\section{Results}

\section{Baseline characteristics}

The characteristics of the whole QUOVADIS sample at baseline have been described in detail in previous reports (Dalle Grave et al., 2004; Melchionda et al., 2003).
The present study sample comprises 500 patients ( 394 females and 106 males) who completed the 1-year follow-up among the 1530 Caucasian patients enrolled by the 18 centers where a control was programmed (Table 1 ). The study was characterized by a high drop-out rate, due both to drop-out of centers (seven centers did not participate in the 12 months follow-up) and to drop-out within centers. The completers differed from the dropouts in a few features. They were nearly 3-year younger $(M=43.7$ years, $S D=11.0$ in completers vs. $M=46.4$ years, $S D=10.8$ in drop-outs; $t(1,1528)=-4.47, p<0.01)$, had a higher BES score $(M=15.3, \mathrm{SD}=9.4$ vs. $M=13.9, \mathrm{SD}=9.4 ; t(1,1509)=2.73$, $p<0.05)$ and a higher BMI $\left(M=38.2 \mathrm{~kg} / \mathrm{m}^{2}, \mathrm{SD}=6.4\right.$ vs. $M=37.3, \mathrm{SD}=5.9 ; t(1,1517)=2.57, p<0.05)$, without differences in SCL-GSI.

Within the population available at follow-up, females had significantly higher mean BES and SCL-GSI scores than males, and a higher prevalence of BES and SCL-GSI scores over the tentative cutoffs of psychopathology.

\section{2-Month changes}

The mean percentage weight loss was similar in males $(9.0 \%$, $\mathrm{SD}=7.7$ ) and females $(7.6 \%, \mathrm{SD}=8.5, t(1,490)=1.45 ; p=\mathrm{ns})$, and $67.9 \%$ of males and $59.9 \%$ of females achieved a weight loss larger than $5 \%\left(\chi^{2}(1,490)=2.35, p=0.126\right)$. There was no significant group effect of BMI (Table 2 ).

For BES, the main effects of Time and Group were significant. This indicated that, after 12 months, the score of BES was significantly reduced in both groups, but females maintained a significantly higher BES score than males both at baseline and after 12 months. The BES score showed a significant Time $\times$ Group interaction, indicating that females had a larger BES improvement $(M=3.7, \mathrm{SD}=7.9)$ than males $(M=2.0, \mathrm{SD}=5.9, t(1,490)=-2.46$; $p=0.015$ ) (Table 2).

Table 1

Baseline data of patients included in the analysis. Data are reported as means (SD) or as \% of cases.

\begin{tabular}{|c|c|c|c|c|c|}
\hline & Females $(N=394)$ & Males $(N=106)$ & $F(\mathrm{df})$ & $\chi^{2}(\mathrm{df})$ & $p$-Value ${ }^{a}$ \\
\hline \multicolumn{6}{|l|}{ Demographic variables } \\
\hline Age (years) & $46.2(10.8)$ & $47.1(10.8)$ & $0.58(1,498)$ & & 0.445 \\
\hline Body Mass Index $\left(\mathrm{kg} / \mathrm{m}^{2}\right)$ & $37.3(5.6)$ & $37.2(6.9)$ & $0.02(1,498)$ & & 0.895 \\
\hline SCL-90 Global Severity Index & $0.82(0.60)$ & $0.54(0.41)$ & $-5.56(1,498)$ & & $<0.001$ \\
\hline Normal $(<1.00)(\%)$ & 68.3 & 84.9 & & & \\
\hline Mild distress (1.00-1.49)(\%) & 16.2 & 9.4 & & $13.76(3)$ & 0.003 \\
\hline Moderate distress (1.50-1.99)(\%) & 9.1 & 5.7 & & & \\
\hline Severe distress $(\geq 2.00)(\%)$ & 6.3 & 0 & & & \\
\hline Binge Eating Scale & $15.0(9.6)$ & $10.0(7.5)$ & $23.72(1,498)$ & & $<0.001$ \\
\hline Score > $16(\%)$ & 37.3 & 18.9 & & $12.77(1)$ & $<0.001$ \\
\hline Score $>26(\%)$ & 15.2 & 3.8 & & $0.82(1)$ & 0.002 \\
\hline
\end{tabular}

SCL-90 = Symptom CheckList-90.

a ANOVA or Chi-square test.

Table 2

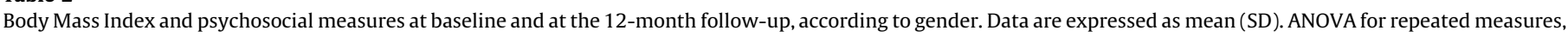
as well as effect sizes, are also reported.

\begin{tabular}{|c|c|c|c|c|c|c|c|}
\hline & \multirow[t]{2}{*}{ Gender } & \multirow[t]{2}{*}{ Baseline } & \multirow[t]{2}{*}{ 12-Month } & \multirow[t]{2}{*}{ Effect size (Cohen's D) } & \multicolumn{3}{|c|}{ ANOVA for repeated measures $F(1,490)$} \\
\hline & & & & & Time & Group & Group $\times$ Time \\
\hline \multirow[t]{2}{*}{ Body Mass Index } & $\mathrm{F}$ & $37.3(5.6)$ & $34.4(5.9)$ & 0.66 & \multirow[t]{2}{*}{$295.76^{*}$} & \multirow[t]{2}{*}{0.38} & \multirow[t]{2}{*}{2.32} \\
\hline & M & $37.2(6.9)$ & $33.8(6.2)$ & 0.70 & & & \\
\hline \multirow[t]{2}{*}{ SCL-GSI } & $\mathrm{F}$ & $0.82(0.60)$ & $0.71(0.59)$ & 0.25 & \multirow[t]{2}{*}{$17.07^{*}$} & \multirow[t]{2}{*}{$20.92^{*}$} & \multirow[t]{2}{*}{0.56} \\
\hline & M & $0.54(0.41)$ & $0.46(0.43)$ & 0.21 & & & \\
\hline \multirow[t]{2}{*}{ Binge Eating Scale } & $\mathrm{F}$ & $15.0(9.6)$ & $11.2(8.6)$ & 0.43 & \multirow[t]{2}{*}{$48.06^{*}$} & \multirow[t]{2}{*}{$20.80^{*}$} & \multirow[t]{2}{*}{$4.37^{*}$} \\
\hline & M & $10.0(7.5)$ & $8.1(6.9)$ & 0.32 & & & \\
\hline
\end{tabular}

Note. $\mathrm{F}=$ females $(N=394) ; \mathrm{M}=$ males $(N=106)$; SCL-GSI = Symptom CheckList-90 Global Severity Index. $p<0.001$. 
Table 3

Pearson's correlations between the anthropometric and clinical variables and psychological measures.

\begin{tabular}{lcccccc}
\hline & Age & BMI & BES & SCL-GSI & $\Delta$ BMI & $\Delta$ BES \\
\hline Age & - & & & & & \\
BMI & -0.03 & - & & & & \\
BES & -0.01 & $0.11^{*}$ & - & & & \\
SCL-GSI & 0.09 & 0.08 & $0.52^{* *}$ & - & & \\
$\Delta$ BMI & $-0.15^{* *}$ & $0.26^{* *}$ & -0.07 & -0.08 & - & \\
$\Delta$ BES & $-0.10^{*}$ & 0.01 & $0.53^{* *}$ & $0.23^{* *}$ & $0.15^{* *}$ & - \\
$\Delta$ SCL-GSI & -0.05 & -0.05 & 0.08 & $0.38^{* *}$ & $0.12^{* *}$ & $0.34^{* *}$ \\
\hline
\end{tabular}

$p<0.05$.

$p<0.01$.

For SCL-GSI, the main effects of Time and Group were significant. This indicated that at follow-up the score of SCL-GSI was significantly reduced in both groups, but females maintained a significantly higher SCL-GSI score than males both at baseline and after 12 months. Contrary to BES, there was no Time $\times$ Group interaction (Table 2).

Finally, the patients who achieved a weight loss $\geq 5 \%$ ( $61.8 \%$ of total sample) showed a larger reduction in SCL-GSI score $(M=0.14$, $\mathrm{SD}=0.44)$ and $\mathrm{BES}$ score $(M=4.2, \mathrm{SD}=7.8)$ compared with subjects who did not get this weight loss outcome (SCL-GSI: $M=0.04, \mathrm{SD}=0.41 ; t(1,490)=-2.40, p=0.017 ; \mathrm{BES}: M=1.8$, $\mathrm{SD}=7.0 ; t(1,490)=-3.44, p=0.001$, respectively $)$.

In general, correlations were demonstrated between clinical and anthropometric variables and psychological measures at baseline and their changes as the effect of the weight loss intervention (Table 3).

Association of psychological distress and binge eating changes with clinical characteristics

Linear regression analysis on the whole sample showed that the reduced SCL-GSI score was significantly associated with BMI changes, which accounted for $17 \%$ of the variance of changes in psychological distress, after adjustment for gender, initial BMI, age and center variability. Similarly, the reduced BES score was significantly associated with reduced BMI, accounting for $20 \%$ of the variance of changes in binge eating (Table 4). Similar results were obtained when females and males were analyzed separately (not reported in details), and, in both groups, the larger the weight

\section{Table 4}

Predictors of changes in the Symptom CheckList-90 Global Severity Index ( $\Delta$ SCLGSI) and the Binge Eating Scale ( $\Delta$ BES), derived from linear regression analysis. All data were adjusted for gender, initial BMI and center variability.

\begin{tabular}{|c|c|c|c|c|}
\hline \multirow{2}{*}{$\begin{array}{l}\text { Model 1: Dependent variable, } \\
\Delta \text { SCL-GSI }^{\mathrm{a}}\end{array}$} & \multicolumn{4}{|c|}{ Parameter } \\
\hline & $\beta$ & SE $\beta$ & $T$ & $p$ \\
\hline BMI changes & 0.13 & 0.01 & 2.85 & 0.005 \\
\hline Age & -0.00 & 0.00 & -0.55 & 0.584 \\
\hline BMI & -0.07 & 0.00 & -1.56 & 0.118 \\
\hline Gender & 0.04 & 0.05 & 0.87 & 0.384 \\
\hline Center variability & 0.08 & 0.00 & 1.72 & 0.085 \\
\hline \multirow{2}{*}{$\begin{array}{l}\text { Model 2: Dependent variable, } \\
\Delta \mathrm{BES}^{\mathrm{b}}\end{array}$} & \multicolumn{4}{|c|}{ Parameter } \\
\hline & $\bar{\beta}$ & $\operatorname{SE} \beta$ & $T$ & $p$ \\
\hline BMI changes & 0.15 & 0.11 & 3.21 & 0.001 \\
\hline Age & -0.07 & 0.03 & -1.60 & 0.109 \\
\hline BMI & -0.03 & 0.06 & -0.58 & 0.559 \\
\hline Gender & 0.10 & 0.84 & 2.26 & 0.024 \\
\hline Center variability & 0.00 & 0.05 & -0.20 & 0.842 \\
\hline
\end{tabular}

${ }^{a}$ Regression model: $F(5,481)=3.02, p=0.011$. The inclusion of BES at baseline and $\triangle \mathrm{BES}$ as confounders did not change the results.

b Regression model: $F(5,473)=3.91, p=0.002$. The inclusion of SCL-GSI at baseline and $\Delta$ SCL-GSI as confounders did not change the results. loss, the better the improvement in psychological variables. Also the inclusion of other psychopathological variables in the regression models (BES and SCL-GSI scores) did not change the significance.

In all models the Variance Inflation Factor was $<2$, indicating the absence of multicolinearity.

\section{Discussion}

The principal finding of the study is that weight loss is associated with reduced psychological distress and binge eating at 12-month follow-up in the "real world" of patients with obesity seeking treatment at medical centers. The beneficial effects were not related to any specific treatment for their psychological distress and binge eating at the time of their first visit.

At baseline, BMI and age were not different between males and females, but females, had higher levels of psychological distress and binge eating. In our sample about $30 \%$ of obese females and $15 \%$ of obese males seeking treatment reported psychological distress and binge eating. These figures are similar to those observed in previous studies based on self-reported questionnaires (de Zwaan, 2001; Spitzer et al., 1991, 1993).

At 12-month follow-up, the moderate percent weight loss ( $9 \%$ in males and $7.6 \%$ in females) was expected to reduce the severity of obesity-associated risk factors (i.e., hypertension, high plasma triglycerides, low plasma HDL-cholesterol, high plasma fasting glucose) (National Institutes of Health, 1998), and produced a significant improvement in both psychological distress and binge eating. The average effect was larger for binge eating, with an effect size of 0.43 in females and 0.32 in males, classified as medium (Kazis et al., 1989), compared to small for psychological distress (0.25 and 0.21 , respectively).

Linear regression analysis found that the amount of weight loss was positively associated with improved psychological distress and binge eating in patients who remained in follow-up. Unfortunately, the study was only aimed at evaluating the association between variables and we cannot make any inference on causality between changes in body weight and changes in psychological factors.

Our findings have clinical implications. Clinicians and health care providers should be reassured that dieting and moderate weight loss is not associated with negative emotional consequences, but with a significant improvement of psychological distress and binge eating in participants compliant to follow-up.

The study has some weaknesses. First, binge eating and psychological distress have been evaluated with self-reported questionnaires, instruments that tend to overestimate the prevalence of psychopathology (Fairburn \& Beglin, 1994). Secondly, only $33 \%$ of the sample was available at follow-up, limiting the external validity of our findings. At baseline, completers differed from dropouts only in a few clinical and psychological variables tested at post hoc analysis, and no inference can be made regarding the total sample of treatment-seeking obese people. In particular, we do not know whether the planned weight loss program had any negative effect on the psychological status and binge eating of the participants who dropped out. The high attrition rate in our sample underlines the difficulties of any long-term evaluation of outcomes in the area of the management of obesity in the real world. Thirdly, our study was not designed to evaluate whether improved binge eating and psychological distress are the result of other unspecific factors not assessed in the present study (e.g., the quality of the therapeutic relationship, or simply attending a medical center over the course of 12 months and being cared for by therapists). We controlled our results for center variability, but the large heterogeneity of treatment procedures between centers and their sequential use during the 1 -year follow-up made a per 
treatment analysis impossible. This issue can only be assessed by ad hoc randomized controlled trials. Fourthly, not all measures were obtained in fasting participants and small changes in body weight might have been missed.

Future studies should investigate the mechanism(s) through which weight loss treatment improves binge eating and psychological distress and whether the psychological benefits will be maintained in the participants who regain weight, a common longterm outcome of obesity treatment (Garner \& Wooley, 1991). At present, the observation that adding cognitive procedures to dietetic treatments is associated with less relapse (Stahre \& Hallstrom, 2005; Stahre, Tarnell, Hakanson, \& Hallstrom, 2007; Werrij et al., 2008, 2009) should encourage the adoption of this combined treatment to maintain the improvement of binge eating and psychological distress associated with weight loss.

\section{Acknowledgement}

The QUOVADIS study was supported by an unrestricted grant from BRACCO Imaging Spa, Milan, Italy.

\section{References}

Agras, W. S., Telch, C. F., Arnow, B., Eldredge, K. L., Wilfley, D., Raeburn, S. D., et al. (1994). Weight loss, cognitive-behavioral and desimipramine treatments in binge eating disorder: an additive design. Behaviour Therapy, 25, 225-238.

Brownell, K. D., \& Stunkard, A. J. (1981). Couples training, pharmacotherapy, and behavior therapy in the treatment of obesity. Archives of General Psychiatry, 38, 1224-1229.

Bryan, J., \& Tiggemann, M. (2001). The effect of weight-loss dieting on cognitive performance and psychological well-being in overweight women. Appetite, 36, $147-156$.

Butryn, M. L., \& Wadden, T. A. (2005). Treatment of overweight in children and adolescents: does dieting increase the risk of eating disorders? International Journal of Eating Disorders, 37, 285-293.

Cohen, J. (1977). Statistical power analysis for the behavioural sciences. New York: Academic Press.

Dalle Grave, R., Calugi, S., Magri, F., Cuzzolaro, M., Dall'Aglio, E., Lucchin, L., et al. (2004). Weight loss expectations in obese patients seeking treatment at medical centers. Obesity Research, 12, 2005-2012.

Dalle Grave, R., Calugi, S., Molinari, E., Petroni, M. L., Bondi, M., Compare, A., et al. (2005). Weight loss expectations in obese patients and treatment attrition: an observational multicenter study. Obesity Research, 13, 1961-1969.

Delinsky, S. S., Latner, J. D., \& Wilson, G. T. (2006). Binge eating and weight loss in a selfhelp behavior modification program. Obesity (Silver Spring), 14, 1244-1249.

Derogatis, L. R., \& Cleary, P. A. (1977). Confirmation of the dimensional structure of the SCL-90: a study in construct validity. Journal of Clinical Psychology, 33, 981-989.

de Zwaan, M. (2001). Binge eating disorder and obesity. International Journal of Obesity and Related Metabolic Disorders, 25(Suppl. 1), S51-S55.

Fairburn, C. G., \& Beglin, S. J. (1994). Assessment of eating disorders: interview or selfreport questionnaire? International Journal of Eating Disorders, 16, 363-370.

Garner, D. M., \& Wooley, S. C. (1991). Confronting the failure of behavioral and dietary treatments for obesity. Clinical Psychology Review, 11, 729-780.

Golay, A., Laurent-Jaccard, A., Habicht, F., Gachoud, J. P., Chabloz, M., Kammer, A., et al. (2005). Effect of orlistat in obese patients with binge eating disorder. Obesity Research, 13, 1701-1708.

Gorin, A. A., Niemeier, H. M., Hogan, P., Coday, M., Davis, C., DiLillo, V. G., et al. (2008) Binge eating and weight loss outcomes in overweight and obese individuals with type 2 diabetes: results from the Look AHEAD trial. Archives of General Psychiatry, $65,1447-1455$

Gormally, J., Block, S., Daston, S., \& Rardin, D. (1982). The assessment of binge eating severity among obese persons. Addictive Behavior, 7, 47-55.

Greeno, C. G., Marcus, M. D., \& Wing, R. R. (1995). Diagnosis of binge eating disorder: discrepancies between a questionnaire and clinical interview. International Journal of Eating Disorders, 17, 153-160.

Kazis, L. E., Anderson, J. J., \& Meenan, R. F. (1989). Effect sizes for interpreting changes in health status. Medical Care, 27, S178-S189.

Kiortsis, D. N., Tsouli, S., Filippatos, T. D., Konitsiotis, S., \& Elisaf, M. S. (2008). Effects of sibutramine and orlistat on mood in obese and overweight subjects: a randomised study. Nutrition Metabolism \& Cardiovascular Disorders, 18, 207-210.

Melchionda, N., Marchesini, G., Apolone, G., Cuzzolaro, M., Mannucci, E., Grossi, E., et al. (2003). The OUOVADIS study. Features of obese Italian patients seeking treatment at specialist centers. Diabetes Nutrition \& Metabolism, 16, 115-124.

National Institutes of Health. (1998). Clinical guidelines on the identification, evaluation, and treatment of overweight and obesity in adults-the evidence report Obesity Research, 6(Suppl. 2), 51S-209S.

National Task Force on the Prevention and Treatment of Obesity. (2000). Dieting and the development of eating disorders in overweight and obese adults. Archives of Internal Medicine, 160, 2581-2589.
Porzelius, L. K., Houston, C., Smith, M., Arkin, C., \& Fisher, E. (1995). Comparison of standard weight loss treatment and a binge eating weight loss treatment. Behaviour Therapy, 26, 199-234.

Presnell, K., \& Stice, E. (2003). An experimental test of the effect of weight-loss dieting on bulimic pathology: tipping the scales in a different direction. Journal of Abnormal Psychology, 112, 166-170.

Raymond, N. C., de Zwaan, M., Mitchell, J. E., Ackard, D., \& Thuras, P. (2002). Effect of a very low calorie diet on the diagnostic category of individuals with binge eating disorder. International Journal of Eating Disorders, 31, 49-56.

Sherwood, N. E., Jeffery, R. W., \& Wing, R. R. (1999). Binge status as a predictor of weight loss treatment outcome. International Journal of Obesity and Related Metabolic Disorders, 23, 485-493.

Spitzer, R. L., Devlin, M., Walsh, B. T., Hasin, D., Wing, R., Marcus, M. D., et al. (1991). Binge eating disorder: a multisided field trial of the diagnostic criteria. International Journal of Eating Disorders, 1, 191-203.

Spitzer, R. L., Yanovski, S., Wadden, T., Wing, R., Marcus, M. D., Stunkard, A., et al. (1993). Binge eating disorder: its further validation in a multisite study. International Journal of Eating Disorders, 13, 137-153.

Stahre, L., \& Hallstrom, T. (2005). A short-term cognitive group treatment program gives substantial weight reduction up to 18 months from the end of treatment. A randomized controlled trial. Eating $\mathcal{E}$ 'Weight Disorders, 10, 51-58.

Stahre, L., Tarnell, B., Hakanson, C. E., \& Hallstrom, T. (2007). A randomized controlled trial of two weight-reducing short-term group treatment programs for obesity with an 18-month follow-up. International Journal of Behavioral Medicine, 14, 4855.

Stice, E., Marti, C. N., Spoor, S., Presnell, K., \& Shaw, H. (2008). Dissonance and healthy weight eating disorder prevention programs: long-term effects from a randomized efficacy trial. Journal of Consulting and Clinical Psychology, 76, 329-340.

Stice, E., Martinez, E. E., Presnell, K., \& Groesz, L. M. (2006). Relation of successful dietary restriction to change in bulimic symptoms: a prospective study of adolescent girls. Health Psychology, 25, 274-281.

Stunkard, A. J., \& Rush, J. (1974). Dieting and depression re-examined: a critical review of reports of untoward responses during weight reduction for obesity. Annals of Internal Medicine, 81, 526-533.

Taylor, C. B., Ferguson, J. M., \& Reading, J. C. (1978). Gradual weight loss and depression. Behaviour Therapy, 9, 622-625.

Teixeira, P. J., Going, S. B., Sardinha, L. B., \& Lohman, T. G. (2005). A review of psychosocial pre-treatment predictors of weight control. Obesity Reviews, 6, 43-65.

Telch, C. F., \& Agras, W. S. (1993). The effects of very-low calorie diet on binge eating. Behaviour Therapy, 24, 177-193.

Wadden, T. A., Berkowitz, R. I., Vogt, R. A., Steen, S. N., Stunkard, A. J., \& Foster, G. D. (1997). Lifestyle modification in the pharmacologic treatment of obesity: a pilot investigation of a potential primary care approach. Obesity Research, 5, 218-226.

Wadden, T. A., Foster, G. D. \& Letizia, K. A. (1994). One-year behavioral treatment of obesity: comparison of moderate and severe caloric restriction and the effects of weight maintenance therapy. Journal of Consulting and Clinical Psychology, 62, 165171

Wadden, T. A., Foster, G. D., Sarwer, D. B., Anderson, D. A., Gladis, M., Sanderson, R. S., et al. (2004). Dieting and the development of eating disorders in obese women: results of a randomized controlled trial. American Journal of Clinical Nutrition, 80, 560-568.

Wadden, T. A., \& Stunkard, A. J. (1986). Controlled trial of very low calorie diet, behavior therapy, and their combination in the treatment of obesity. Journal of Consulting and Clinical Psychology, 54, 482-488.

Wadden, T. A., Stunkard, A. J., Brownell, K. D., \& Day, S. C. (1985). A comparison of two very-low-calorie diets: protein-sparing-modified fast versus protein-formula-liquid diet. American Journal of Clinical Nutrition, 41, 533-539.

Werrij, M. Q., Jansen, A., Mulkens, S., Elgersma, H. J., Ament, A. J., \& Hospers, H. J. (2009). Adding cognitive therapy to dietetic treatment associated with less relapse in obesity. Journal of Psychosomatic Research, 67, 315-324.

Werrij, M. Q., Mulkens, S., Hospers, H. J., Smits-de Bruyn, Y., \& Jansen, A. (2008). Dietary treatment for obesity reduces BMI and improves eating psychopathology, selfesteem, and mood. Netherlands Journal of Psychology, 64, 8-14.

Wilfley, D. E., Crow, S. J., Hudson, J. I., Mitchell, J. E., Berkowitz, R. I., Blakesley, V., et al. (2008). Efficacy of sibutramine for the treatment of binge eating disorder: a randomized multicenter placebo-controlled double-blind study. American Journal of Psychiatry, 165, 51-58.

Wing, R., Marcus, B. H., Epstein, L. H., Blair, S. N., \& Burton, L. R. (1989). Binge eating in obese patients with type 2 diabetes. International Journal of Eating Disorders, 8, 671-679.

Wing, R. R., Blair, E., Marcus, M., Epstein, L. H., \& Harvey, J. (1994). Year-long weight loss treatment for obese patients with type II diabetes: does including an intermittent very-low-calorie diet improve outcome? American Journal of Medicine, 97, 354362 .

Wing, R. R., Marcus, M. D., Blair, E. H., \& Burton, L. R. (1991). Psychological responses of obese type II diabetic subjects to very-low-calorie diet. Diabetes Care, 14, 596599.

Wing, R. R., Marcus, M. D., Epstein, L. H., \& Kupfer, D. (1983). Mood and weight loss in a behavioral treatment program. Journal of Consulting and Clinical Psychology, 51, 153-155.

Yanovski, S. Z. (2003). Binge eating disorder and obesity in 2003: could treating an eating disorder have a positive effect on the obesity epidemic? International Journal of Eating Disorders, 34(Suppl.), S117-S120.

Yanovski, S. Z., Gormally, J. F., Leser, M. S., Gwirtsman, H. E., \& Yanovski, J. A. (1994). Binge eating disorder affects outcome of comprehensive very-low-calorie diet treatment. Obesity Research, 2, 205-212. 